\title{
Competing risks for reliability analysis using Cox's model
}

\begin{abstract}
Purpose - Cox's model with Weibull distribution and Cox's with exponential distribution are the most important models in reliability analysis. This paper seeks to show that, with a large sample size based on expectation maximization (EM) algorithm, both models give similar results. Design/methodology/approach - The parameters of the models have been estimated by method of maximum likelihood based on EM algorithm. The objective of this analysis is to fit the modification of Cox's model with Weibull distribution and Cox's with exponential distribution, examine its performance and compare their results with Crowder et al. Findings - A simulation study indicates that the parametric Cox's model with Weibull distribution gives similar results to Cox's with exponential distribution, especially for a large sample size. Also, the modification of the two models showed better results compared with Crowder et al., especially for the second causes of failure. Originality/value - A modification of the two competing risk models has mostly been applied in failure time data and simulation data. The results of the simulation study indicate that the Weibull and exponential are suitable for Cox's model as they are easy to use and it can achieve even higher accuracy compared with other distribution models.
\end{abstract}

Keyword: Modelling, Algorithmic languages, Exponential distribution, Reliability management 